\title{
Establishment of a human lung cancer cell line with high metastatic potential to multiple organs: Gene expression associated with metastatic potential in human lung cancer
}

\author{
TETSUHIRO NAKANO ${ }^{1}$, KIMIHIRO SHIMIZU ${ }^{1}$, OSAMU KAWASHIMA ${ }^{1}$, MITSUHIRO KAMIYOSHIHARA ${ }^{1}$, \\ SEIICHI KAKEGAWA ${ }^{1}$, MASAYUKI SUGANO ${ }^{1}$, TAKASHI IBE ${ }^{1}$, TOSHITERU NAGASHIMA ${ }^{1}$, \\ KYOICHI KAIRA $^{2}$, NORIAKI SUNAGA ${ }^{2}$, YOUICHI OHTAKI ${ }^{1}$, JUN ATSUMI $^{1}$ and IZUMI TAKEYOSHI ${ }^{1}$ \\ Departments of ${ }^{1}$ Thoracic and Visceral Organ Surgery and ${ }^{2}$ Medicine and Molecular Science, \\ Gunma University Graduate School of Medicine, Gunma 371-8511, Japan
}

Received January 18, 2012; Accepted March 7, 2012

DOI: 10.3892/or.2012.1972

\begin{abstract}
Convenient and reliable multiple organ metastasis model systems might contribute to understanding the mechanism(s) of metastasis of lung cancer, which may lead to overcoming metastasis and improvement in the treatment outcome of lung cancer. We isolated a highly metastatic subline, PC14HM, from the human pulmonary adenocarcinoma cell line, PC14, using an in vivo selection method. The expression of 34,580 genes was compared between PC14HM and parental PC14 by cDNA microarray analysis. Among the differentially expressed genes, expression of four genes in human lung cancer tissues and adjacent normal lung tissues were compared using real-time reverse transcription polymerase chain reaction. Although BALB/c nude mice inoculated with parental PC14 cells had few metastases, almost all mice inoculated with PC14HM cells developed metastases in multiple organs, including the lung, bone and adrenal gland, the same progression seen in human lung cancer. cDNA microarray analysis revealed that 981 genes were differentially (more than 3-fold) expressed between the two cell lines. Functional classification revealed that many of those genes were associated with cell growth, cell communication, development and transcription. Expression of three upregulated genes (HRB-2, HS3ST3Al and $R A B 7$ ) was higher in human cancer tissue compared to normal lung tissue, while expression of $E D G l$, which was downregulated, was lower in the cancer tissue compared to the normal lung. These results suggest that the newly established PC14HM cell line may provide a mouse model of widespread
\end{abstract}

Correspondence to: Dr Kimihiro Shimizu, Department of Thoracic and Visceral Organ Surgery, Gunma University Graduate School of Medicine, 3-39-15 Showa-machi Maebashi, Gunma 371-8511, Japan

E-mail:kmshimizu@gmail.com

Key words: lung cancer, mouse model, metastasis, multiple organ, microarray metastasis of lung cancer. This model system may provide insights into the key genetic determinants of widespread metastasis of lung cancer.

\section{Introduction}

Lung cancer is a leading cause of cancer-related death worldwide (1). The 5-year survival rate for patients with non-small cell lung cancer (NSCLC) is less than 50\% (2). The development of widespread distant metastases after initial surgery is the main reason for cancer-related deaths in operable NSCLC. Overcoming widespread metastases might contribute to a marked improvement in the treatment outcome of lung cancer. Understanding the mechanism(s) of metastasis of lung cancer is indispensable for the development of novel treatment strategies.

It is thought that development of distant metastases requires a multi-step process, such as invading the surrounding tissue, entering the lymphatic or blood vessels, surviving in the circulation, extravasating into a new tissue, and growing in the new organ (3). Lung cancers can metastasize to a variety of organs, but like other cancers, there is an organ preference for metastatic spread in lung cancer. The organ-preference patterns of tumor metastasis have been proposed to be the product of favorable interactions between metastatic tumor cells the 'seed' and the organ microenvironment the 'soil' (4).

To date, part of the physics and biology of cancer metastasis has been examined and several molecules associated with metastasis have been identified and shown to play key roles in metastasis (5). However, the molecular mechanisms of metastasis in lung cancer remain unresolved.

Precise analyses of the pathological, biological, molecular, and genetic aspects of tumor metastasis using tissue specimens from metastatic organs might contribute to further understanding of the mechanism of metastasis and to the development of new treatment modalities. However, it is much more difficult to obtain tissue specimens from metastatic organs than from primary tumors, not least because surgery is not usually the standard treatment for metastasis of various cancers. Furthermore, preclinical therapeutic studies on 
new treatment modalities are needed before clinical trials in patients with advanced metastatic disease.

Several mouse models of metastasis have been developed for various cancers (6-9). However, the majority of human lung cancer cell lines do not have the ability to metastasize to multiple organs beyond the lung in conventional athymic nude mice, even though the models were developed by directly injecting human cancer cells into the vessels of these nude mice (10). One way to address this is to establish human cancer cell lines with a robust ability to metastasize to multiple organs in conventional nude mice $(7,8,11)$. Such highly metastatic cell lines might provide mouse metastasis models without troublesome manipulation and would be particularly suitable for comparative studies on biological and molecular mechanisms of metastasis using subclones with differing metastatic potential.

Important data on the molecular mechanisms of metastasis in cancers have been produced from comparative studies of biological behavior and genetic status among clonally related cell lines with different metastatic potentials (11-15). Several genes associated with metastasis have been identified by comparing the gene expression in cells with different metastatic potentials, indicating that this strategy is useful for determining the molecular mechanisms of metastasis $(12,16,17)$. However, the metastatic potential of these cell lines is practically restricted to the lung in mouse models. Cell lines with high metastatic potential to multiple organs would be more appropriate for comparative studies, because such cell lines would more closely reflect the features of human cancer metastasis.

In this study, to develop a convenient and reliable multiple organ metastasis model system, we established a highly metastatic subline, PC14HM, from the PC14 human lung adenocarcinoma cell line using an in vivo method. The highly metastatic subline had the ability to metastasize to multiple organs. To search for genetic determinants of metastasis in human lung cancer, we compared gene expression profiles between the new subline and the parent cells using microarray and real-time reverse transcription polymerase chain reaction (RT-PCR) analyses.

\section{Materials and methods}

Human lung cancer cell lines. Human lung cancer PC14 cells were maintained in RPMI-1640 medium (Sigma, St. Louis, MO), supplemented with $10 \%$ heat-inactivated fetal bovine serum (Life Technologies, Rockville, MD), penicillin, and streptomycin in a humidified atmosphere of $5 \% \mathrm{CO}_{2}$ and $95 \%$ air at $37^{\circ} \mathrm{C}$.

Mice. Female BALB/c nude mice (5 weeks old) were obtained from Charles River Laboratories Japan, Inc. (Tokyo, Japan). Sterile food and water were available to mice ad libitum. The mice were maintained in sterile cages on sterile bedding and housed in rooms at constant temperature and humidity.

Experimental metastasis. Human lung cancer cell lines were injected intravenously (i.v.) into the lateral tail vein of nude mice at a density of $2 \times 10^{6}$ cells $/ 0.2 \mathrm{ml}$. Mice were sacrificed when they became moribund, and an autopsy was performed.
Visceral organs were removed and inspected for the presence of metastases.

Selection of the highly metastatic subline. A human lung cancer cell line with high metastatic potential to multiple organs was established by a method previously described (7). Briefly, mice were injected i.v. with the parental PC14 cells and sacrificed when they became moribund. Under sterile conditions, organs with macroscopic metastasis were removed and a metastasized nodule was dissected free of connective tissue and blood clots, and rinsed twice with medium. The nodule was finely minced using sterile scalpel blades in medium, and the cells were seeded in tissue culture dishes. Then, 4-6 weeks later, the cells were reinjected i.v. into nude mice. This process was repeated three times to establish the highly metastatic subline, PC14HM. PC14HM was derived from an adrenal metastasis, which is uncommon, after i.v. injection of parental PC14 cells. The metastasized cells in the bone after reinjection of the first metastatic subline were reinjected again and cancer cells in the metastatic nodule in the bone were selected as the highly metastatic subline, PC14HM.

Tumorigenicity in nude mice. Tumorigenicity was examined by injecting $1 \times 10^{7}$ cells $/ 0.2 \mathrm{ml}$ into the subcutis of the dorsal flanks of nude mice. The tumor size was measured twice a week. Tumor volume (V) was calculated using the formula $\mathrm{V}$ $=1 / 2 \times$ length $\mathrm{x}$ (width $)^{2}$.

Cell proliferation assay. Cells were seeded in a 96-well plate at a density of $1 \times 10^{4}$ cells/well, and the number of cells at each time point was estimated using the TetraColor One Cell Proliferation Assay System (Seikagaku Corp., Tokyo, Japan) according to the manufacturer's protocol. Doubling times of the cells were calculated from the logarithmic phase of the growth curve.

cDNA microarray analysis. Total RNA was extracted using the RNeasy mini-kit (Qiagen, Tokyo, Japan) according to the supplier's protocol. Microarray analysis was performed using a Filgen Array Human 35K (Filgen, Inc., Nagoya, Japan), consisting of 34,580 cDNA fragments of human genes, 8 cDNA fragments of control human housekeeping genes, and 6 cDNA fragments derived from other species arrayed and immobilized. The 34,580 70-mer probes represented 24,650 genes and 37,123 gene transcripts. Fluorescent probes were synthesized by incorporating Cy3- or Cy5-dUTP (Amersham Biosciences, Arlington Heights, IL) using $2 \mu \mathrm{g}$ total RNA as a template and the RNA Transcript SureLABEL Core kit (Takara Bio, Tokyo, Japan), and hybridized to the chip according to the manufacturer's protocol. Imaging and quantifying analyses were performed using the GenePix 4000B scanner (Molecular Devices Japan, Tokyo, Japan) and the Array-Pro Analyzer software (ver. 4.5; Media Cybernetics, Inc., Bethesda, MD). Gene expression was quantified as the ratio of PC14HM to PC14. For a cDNA fragment to be selected as being differentially expressed, it had to be expressed in PC14HM cells at a level at least three times higher or lower than in PC14 cells. These genes were then classified based on the described function. cDNA fragments with $>10$-fold difference in expression between the two cell lines are listed. 
Clinical samples. Surgically resected specimens of lung cancer and matched normal lung tissue were obtained from 36 patients who underwent surgery at Gunma University Hospital between October 2002 and March 2006. The specimens consisted of 28 adenocarcinomas and 8 squamous cell carcinomas. After surgical removal, a portion of each sample was immediately frozen and stored at $-80^{\circ} \mathrm{C}$ until total RNA extraction. Institutional approval was obtained from the institutional review board of Gunma University Hospital. Written informed consent was obtained from the patients.

Quantitative real-time PCR ( $P P C R)$ analysis. Four genes, $H R B-2, H S 3 S T 3 A 1, R A B 7$ and $E D G 1$, were randomly selected for this experiment from the list of differentially expressed genes. $H R B-2, H S 3 S T 3 A 1$ and $R A B 7$ were upregulated in PC14HM cells. Expression of EDGl in PC14HM was approximately 200 times lower than in the parental cells in the microarray analysis. Total RNA was extracted using the RNeasy mini-kit (Qiagen) according to the supplier's protocol. Randomly primed cDNAs were reverse-transcribed from $5 \mu \mathrm{g}$ total RNAs using the SuperScript first-strand synthesis system for RT-PCR (Invitrogen, Carlsbad, CA) according to the supplier's protocol. Quantitative analysis of gene expression was performed using the TaqMan PCR method. PCR primers and TaqMan probes were purchased from Applied Biosystems (Foster City, CA). A cDNA conversion mixture (1 $\mu \mathrm{l})$ was used for PCR amplification. The PCR mixture was prepared using a Quantitect Probe PCR kit (Qiagen) according to the manufacturer's protocol. Amplification was performed on an ABI 7300 Fast Real-Time PCR System (Applied Biosystems) programmed to hold at $50^{\circ} \mathrm{C}$ for $2 \mathrm{~min}$, hold at $95^{\circ} \mathrm{C}$ for $15 \mathrm{~min}$, and complete 45 cycles of $94^{\circ} \mathrm{C}$ for $15 \mathrm{sec}$ and $60^{\circ} \mathrm{C}$ for $30 \mathrm{sec}$. The Sequence Detection software (ver. 1.3.1; Applied Biosystems) was used to measure the quantity of template molecule from the standard curve of the $\mathrm{Ct}$ value for each gene generated from cDNA from human brain poly $(\mathrm{A})^{+} \mathrm{RNA}$ (Clontech, Palo Alto, CA). The level of mRNA expression in human lung cancer specimens for each gene was expressed as the value of the quantity for each gene relative to that for $A C T B$ and compared to that of adjacent normal lung tissues. Each assay was performed in duplicate.

Statistical analysis. Differences in the number of metastatic nodules among each group of mice were analyzed for statistical significance using a Student's t-test. Differences in survival among each group of mice were analyzed for statistical significance using the log-rank test. Differences in the expression of the four selected genes between human NSCLC and normal lung were assessed using a Student's t-test. Differences were considered significant at the $95 \%$ confidence limit. All statistical analyses were conducted using the Statview software (Abacus Concepts Inc., Berkeley, CA).

\section{Results}

Metastatic potential of the highly metastatic subline PC14HM from PC14 cells. To evaluate the metastatic potential of the PC14HM cells, PC14 and PC14HM cells were injected simultaneously into the tail veins of five mice. In the five mice injected with PC14 cells, only one developed macroscopic metastases
Table I. Experimental metastasis of PC14 cells and the subline in $\mathrm{BALB} / \mathrm{c} \mathrm{nu} / \mathrm{nu}$ mice.

\begin{tabular}{lrl}
\hline Cell line & $\begin{array}{c}\text { Survival } \\
\text { (days) }\end{array}$ & \multicolumn{1}{c}{$\begin{array}{c}\text { Experimental } \\
\text { metastasis }\end{array}$} \\
\hline PC14 & 69 & Lung, bone (cranium) \\
(parent) & 96 & ND \\
& 126 & ND \\
& 128 & ND \\
& 130 & ND \\
PC14HM & 19 & Bone \\
& 32 & Lung, bone (pelvis), LN (neck, Md) \\
& 37 & Lung, bone (scapula), Lt. Ad, LN (axilla) \\
& 39 & Bone (pelvis), Lt. Ad \\
& 39 & Lung, bone (thighs, leg), LN (neck)
\end{tabular}

ND, not detected; LN, lymph node; Md, mediastinum; Ad, adrenal gland; Lt, left.
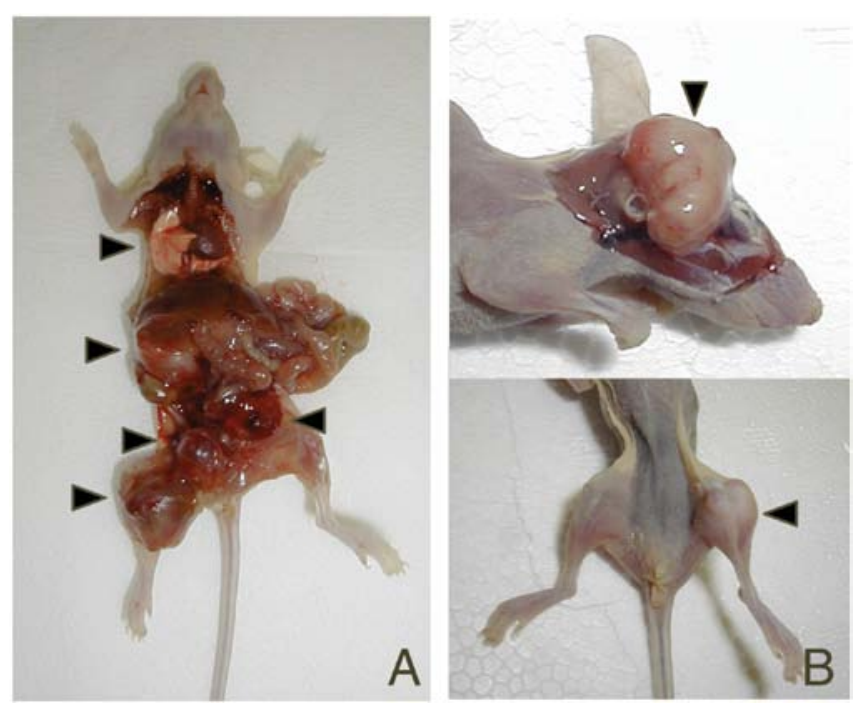

Figure 1. Experimental metastasis of PC14HM cells in BALB/c nu/nu mice. Representative photograph of macroscopic appearance of metastasized organs in mice injected with PC14HM cells. These images were taken when the PC14HM cells were established and this experiment was independent from the experiment shown in Table I. (A) A mouse inoculated with PC14HM cells 41 days before autopsy. Macroscopic metastases (arrowhead) were observed in lung, vertebrae, right thighbone, right adrenal gland, abdominal lymph nodes. (B) A mouse inoculated with PC14HM cells 49 days before autopsy. Metastatic nodules (arrowhead) in cranium and left thighbone were apparent.

in the lung and bone 69 days after injection (Table I). The other four mice were sacrificed between 96 and 130 days after injection. No obvious metastases were observed in these mice. On the other hand, mice injected with PC14HM cells became moribund between 19 and 39 days after injection and autopsies revealed that all five mice developed macroscopic metastatic nodules in the multiple organs, such as the lungs, lymph nodes, bones, and adrenal glands (Table I). The distribution of organs with metastasized cancer was similar to that seen in human lung cancer (Fig. 1). In particular, bone metastases developed in all five mice injected with PC14HM cells. Differences in the 
Table II. Characteristics of PC14 cells and the highly metastatic subline.

Experimental metastasis

\begin{tabular}{lccccc} 
Cell line & $\begin{array}{c}\text { Doubling time } \\
\text { in vitro }(\mathrm{h})\end{array}$ & $\begin{array}{c}\text { In vivo tumor } \\
\text { growth }(\mathrm{days})^{\mathrm{a}}\end{array}$ & Incidence $^{\mathrm{b}}$ & $\begin{array}{c}\text { Metastases/mouse } \\
(\mathrm{n})\end{array}$ & $\begin{array}{c}\text { Survival (days) } \\
\text { (mean } \pm \text { SD) }\end{array}$ \\
\hline PC14 (parent) & 41 & 18.9 & $1 / 5$ & 0.4 & $109.8 \pm 26.7$ \\
PC14HM & 42 & 17.6 & $5 / 6$ & $3.2^{\mathrm{c}}$ & $33.2 \pm 8.4^{\mathrm{d}}$
\end{tabular}

${ }^{\mathrm{a}}$ Average number of days for tumors to reach $1000 \mathrm{~mm}^{3} .{ }^{\mathrm{b}}$ The number of mice with metastases/the number of mice injected. ${ }^{\mathrm{c}}$ The number of metastatic nodules was significantly different than that observed in mice injected with parental $\mathrm{PC} 14$ cells $(\mathrm{P}=0.0101)$. ${ }^{\mathrm{d}}$ Survival periods were significantly shorter than for mice injected with parental PC14 cells $(\mathrm{P}=0.0027)$.

number of metastatic nodules and the survival periods were statistically significant between the highly metastatic subline and the parental cells $(\mathrm{P}=0.0101, \mathrm{P}=0.0027$, respectively; Table II).

Effect of growth rate and tumorigenicity on metastatic ability. The in vitro population doubling times of PC14 and PC14HM were 41 and $42 \mathrm{~h}$, respectively. No statistically significant difference was observed between the cell lines (Table II). To compare tumorigenicity between the two cell lines, the average number of days for tumors to reach $1000 \mathrm{~mm}^{3}$ was compared. The PC14 tumors took 18.9 days to reach $1000 \mathrm{~mm}^{3}$ after subcutaneous injection, whereas the PC14HM tumors took 17.6 days (Table II). There was no statistically significant difference between the cell lines.

Differences in gene expression between PC14 and PC14HM cells. According to the cDNA microarray analysis, $981 \mathrm{cDNAs}$ (among the 34,580 cDNAs) showed 3-fold differences in expression between the PC14 and PC14HM cells (data not shown). The expression of 537 cDNAs was more abundant in PC14HM than in PC14 cells, whereas that of 444 cDNAs was lower in PC14HM than in PC14 cells. Functional classification based on gene ontology biological processes revealed that $>30$ genes
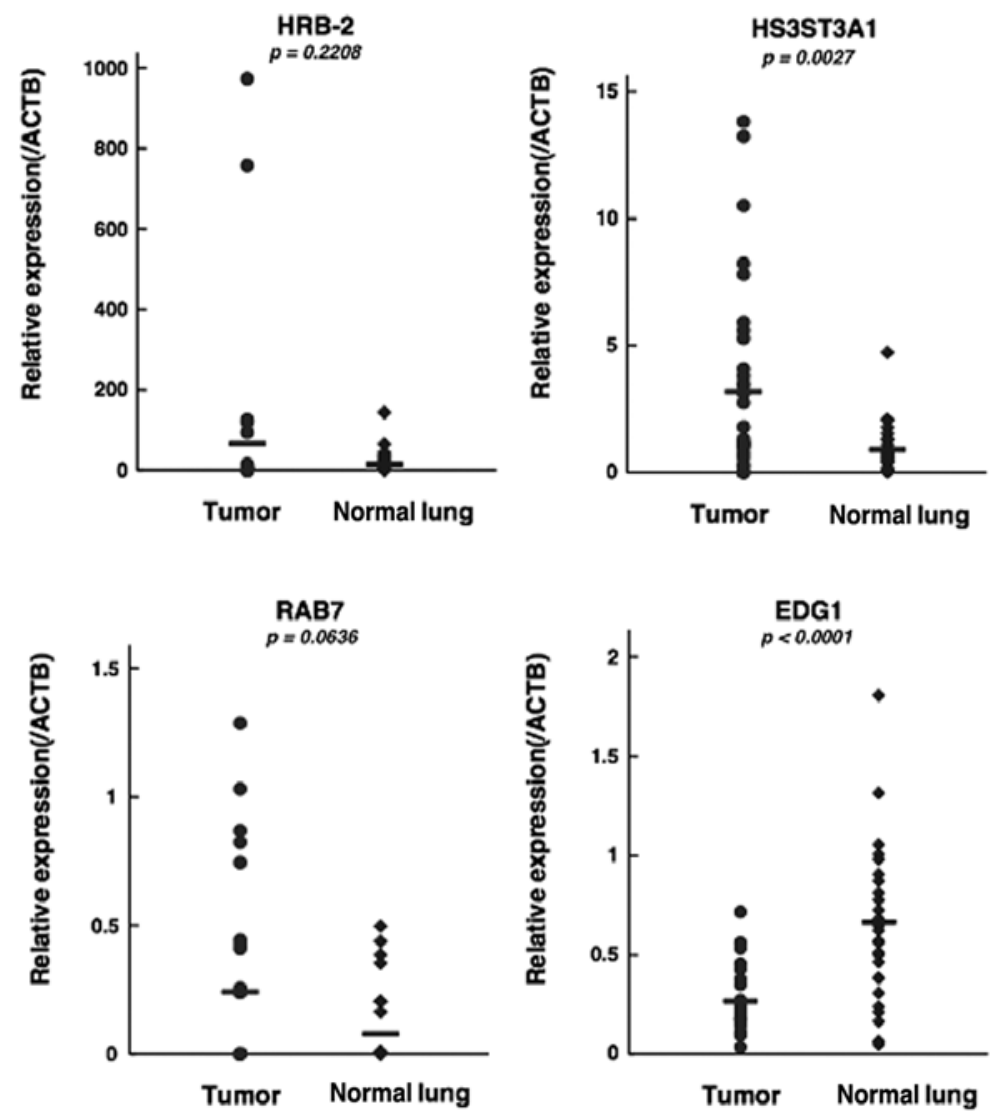

Figure 2. Expression of four genes identified as differentially expressed between PC14 and PC14 HM cells in NSCLC and normal lung tissues. The y-axis represents the ratio of relative expression of each gene to $A C T B$, determined by real-time quantitative RT-PCR analysis. 
Table III. Functional classification of genes differentially expressed between the PC14HM highly metastatic subline and parental PC14 cells.

\begin{tabular}{lcc}
\hline & \multicolumn{2}{c}{ No. of genes } \\
\cline { 2 - 3 } Function & Upregulated & Downregulated \\
\hline Metabolism & 78 & 65 \\
Cell growth and/or & 30 & 29 \\
maintenance & & \\
Cell communication & 23 & 24 \\
Transcription & 21 & 18 \\
Development & 22 & 13 \\
Response to stress & 10 & 13 \\
Signal transduction & 10 & 6 \\
Protein transport & 7 & 4 \\
Antigen processing & 1 & 7 \\
Translation & 5 & 1 \\
Morphogenesis & 4 & 2 \\
Apoptosis & 3 & 3 \\
Cell cycle & 3 & 1 \\
Cell-cell adhesion & 0 & 4 \\
\hline
\end{tabular}

associated with metabolism, cell growth and/or maintenance, cell communication, transcription, and development were contained in the differentially expressed genes (Table III). More than 10-fold differences in expression were found in 28 cDNAs between the two cell lines. Tables IV and V list the genes expressed in PC14HM cells at least 10-fold higher or lower than in PC14 cells, respectively. In listing the genes, two cDNAs without gene information were omitted from the 11 cDNAs that were expressed in PC14HM more abundantly than in PC14 cells. Thus, 9 upregulated genes and 17 downregulated genes are listed in Tables IV and V, respectively.

Expression of genes differentially expressed between PC14 and PC14HM cells in human NSCLC and normal lung tissues. In the qPCR analysis, the mean relative expression of the three genes that were upregulated in PC14HM cells, HRB-2, $H S 3 S T 3 A 1$ and $R A B 7$, was also elevated in human lung cancer compared to normal lung (Fig. 2). Expression of HS3ST3AI between lung cancer and normal lung tissues showed a statistically significant difference ( $\mathrm{P}=0.0027$; Fig. 2$)$. Expression of $E D G 1$, which was downregulated in PC14HM cells, was also reduced in human lung cancer compared to normal lung, and the difference was statistically significant $(\mathrm{P}<0.0001$; Fig. 2).

\section{Discussion}

Metastases of lung cancer often appear in multiple organs. Various organs can be attacked by lung cancer cells, which tend to 'prefer' certain organs over others (18). To understand the mechanisms of metastasis of human lung cancer, it is important to identify the metastatic potential of multiple organs. There is limited information about the metastasis of

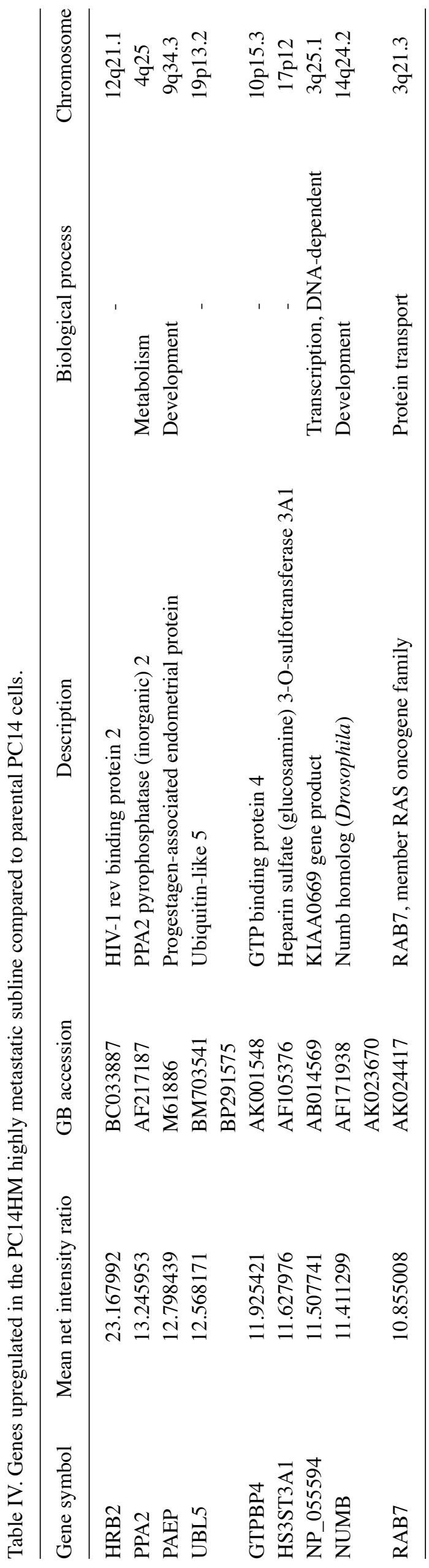




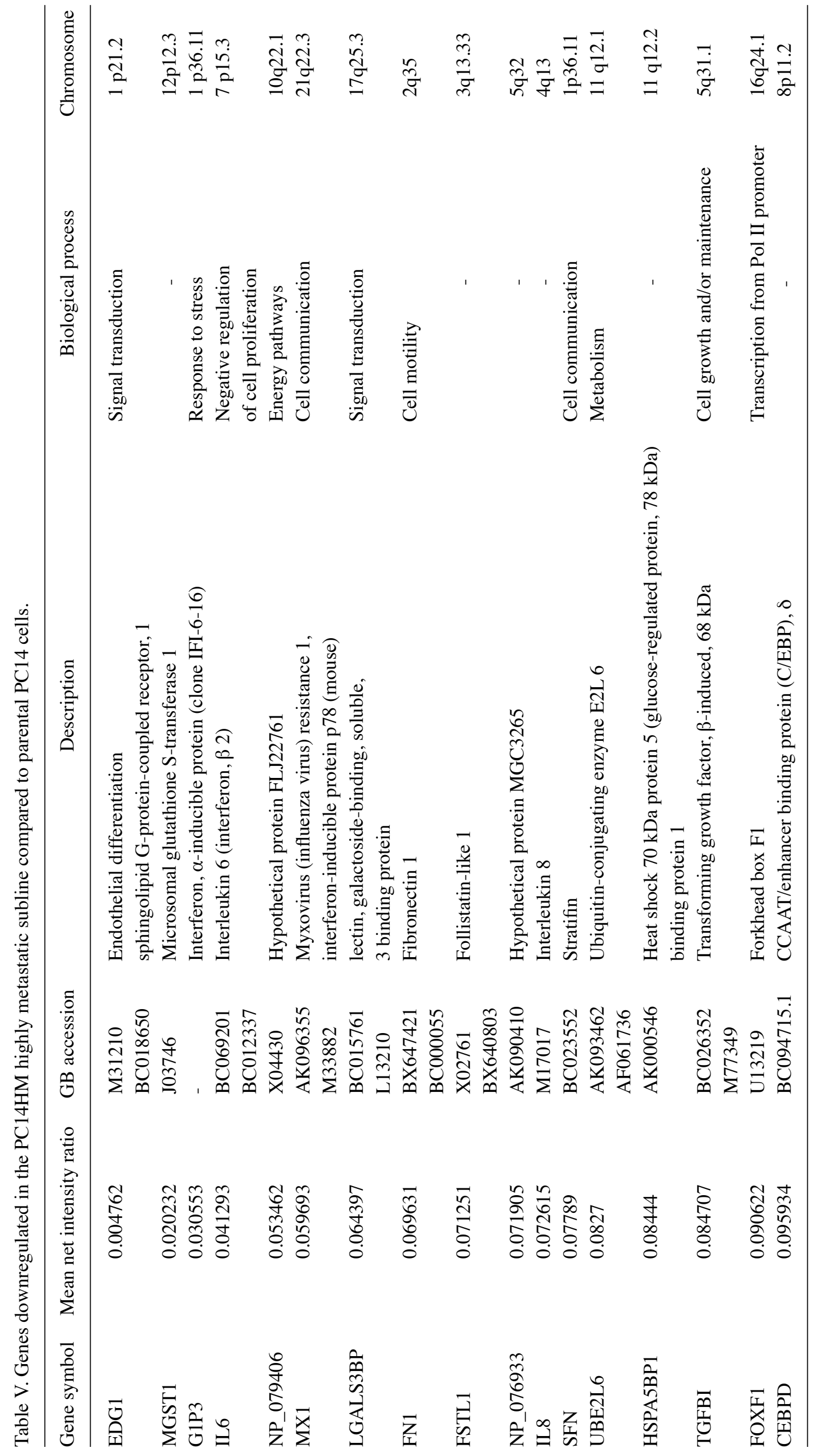


human lung cancer cells for several reasons, including the fact that it is difficult to obtain tissue specimens from multiple metastatic organs and because there is no relevant and simple animal model of the metastasis of human lung cancer.

Until now, the development of animal models for multiple organ metastasis of human lung cancer has required complex immunocompromised host animals, such as severe combined immunodeficient (SCID) mice depleted of natural killer cells with anti-IL-2 receptor $\beta$ chain antibody, or other genetically modified rodents, such as SCID-beige mice or NOD/SCID mice $(6,9,11,19,20)$. To establish a more useful and convenient animal model system for studying metastasis of human lung cancer, we isolated a highly metastatic subline, PC14HM, from the parental PC14 lung adenocarcinoma cell line. Tail vein injection of the highly metastatic subline caused multiple organ metastases in all inoculated nude mice, whereas few metastases were observed after injection of the parental cells. Comparison of gene expression between the highly metastatic subline and their parental cells identified candidate genes that may be involved in regulating the metastatic potential of human lung cancers.

We chose to implant cells via tail vein injection, even though such models generally do not experience several steps of the metastasis process (such as growth in primary organs and invasion into the surrounding tissue and lymphatic or blood vessels) because this method tends to produce a more stable multi-organ metastasis model $(8,9)$. Orthotopically or subcutaneously implanted models show all steps in the metastatic process, but are only rarely successfully produced $(8,9,21)$. There may be several reasons for this; for one, the survival time of mice injected orthotopically or subcutaneously is much shorter than the period it takes tumor cells to metastasize to distant organs, because of the typically rapid growth of primary tumors (9). Subcutaneous inoculation of PC14HM or the parental cells indeed did not cause metastasis when the subcutaneous nodules grew completely.

Mice inoculated with PC14HM cells became moribund within 40 days and developed multiple metastatic nodules (average, 2.8) in multiple organs, such as the lung, bone, adrenal gland and lymph node. This stability in the survival period, incidence, and number of metastases, and the wide variety of distribution of organs with metastases are useful aspects of a multi-organ metastasis model for molecular and genetic studies and for the development of novel treatment strategies of cancer metastasis.

The distribution of metastasized organs in our models was similar to the pattern of metastasis of human lung adenocarcinomas, which is thought to reflect organ heterogeneity and organ 'preferences'. Lung, bone, adrenal gland and lymph nodes are frequent metastasis targets with progression of the disease, and our model system may be useful for studies on organ preferences in the metastatic spread of lung cancer. Bone and brain metastasis especially worsen the patient's quality of life.

Stable development of bone metastases is a distinctive feature of our model. A previous bone metastasis model was reported, but it requires complex manipulations, such as injection of human cancer cell lines into the left ventricle of nude mice (22), while our model system adopts conventional tail-vein injection, a much easier procedure. In the course of developing PC14HM, the subline experienced two cycles of experimental bone metastasis in nude mice. It would be intriguing to investigate whether the distinct bone metastatic ability of PC14HM is the result of a particular organ preference for metastasis to bone.

Brain metastases were not detected macroscopically in any mouse injected with PC14HM cells at the time of autopsy. Conventional experimental metastasis models using severe combined immunodeficient mice also do not develop brain metastases (6). Development of brain metastasis has been reported to require injection of human lung cancer cells into the carotid artery of mice and histopathological conformation of metastatic nodules (23). Thus, to date, research on brain metastasis using mouse models has only been conducted using unique equipment and complex manipulations.

The process of cancer metastasis involves a series of linked sequential steps, including local tumor growth, angiogenesis, invasion, detachment, intravasation, circulation, adhesion, extravasation, and growth in distant organs $(3,8)$. In this study, proliferative activity in vitro and tumorigenicity in vivo showed no significant difference between PC14HM and parental PC14 cells, indicating no obvious correlation between proliferative activity and/or tumorigenicity and metastatic potential of the cell lines. Based on these results, proliferative activity and/ or tumorigenicity may be less important for the regulation of metastatic potential in PC14 cells. Functional studies of clonally related cancer cell lines with different metastatic potentials have demonstrated a relationship between metastatic potential and biological properties other than proliferative activity, such as invasiveness, motility, and adherence $(11,12)$. Further studies on other biological properties of PC14HM and PC14 cells may provide information on important biological determinants regulating the metastatic potential of lung cancer cells.

Cell regulatory pathways that are deregulated in lung cancer include the Rb/p16/cyclin D1, p53/MDM2/p19ARF, Wnt/APC, EGFR/Ras, PP2a, and telomerase pathways (1). PC14 harbors a homozygous deletion of p16 (24) and wildtype $K$-ras and EGFR (25). Activating mutations in the EGFR kinase domain is strongly associated with responsiveness to EGFR kinase inhibitors (26). Also, the presence of these EGFR kinase mutations is associated with improved progression-free survival and overall survival after therapy with EGFR kinase inhibitors, compared to patients with wild-type EGFR (27). Thus, improving the outcome of treatment in patients with metastatic lung cancer harboring wild-type EGFR remains an essential clinical problem to be resolved. Our model system using PC14 may be useful for the assessment of novel therapies against metastatic lung cancer with wild-type EGFR.

To search for genetic determinants for metastasis in PC14 cells, we used a cDNA microarray analysis with the highly metastatic PC14HM cells and their parent cells. Among 34,580 genes examined, 537 upregulated and 444 downregulated genes with at least a 3 -fold difference in expression were identified. Functional classification of these differentially expressed genes demonstrated that more than 30 genes were associated with metabolism, cell growth and/or maintenance, cell communication, transcription, and development. More than 10-fold differences in expression were observed in 26 cDNAs between the two cell lines. In this screen of genes with highly differential expression, multiple genes were associated with the biological processes listed above. It is possible that 
these functional clusters contain genes that are important for understanding and ultimately treating cancer metastases.

To evaluate the expression of the differentially expressed genes in human lung cancer, qPCR analysis using a human lung cancer specimen and adjacent normal lung tissue was performed. We randomly selected four differentially expressed genes, $H R B-2, H S 3 S T 3 A 1, R A B 7$ and $E D G 1$, for qPCR analysis. The expression of three genes, HRB-2, HS3ST3Al and RAB7, upregulated in PC14HM cells, was higher in a human lung cancer specimen than in adjacent normal lung tissue, whereas expression of a downregulated gene, EDG1, was markedly lower in the human lung cancer specimen. Differences in the expression levels of two of the four selected genes, HS3ST3AI and $E D G 1$, were statistically significant. These findings may reflect the functions of these genes in human lung cancer cells, indicating possible associations with not only metastatic potential but also carcinogenesis and progression.

$E D G 1$, also known as SIPRl, encodes a member of the endothelial differentiation gene (Edg) family of proteins, which are $G$ protein-coupled receptors for sphingosine-1-phosphate (S1P), a biologically active metabolite of sphingolipid $(28,29)$. S1P-S1PR1 signaling has been proposed to contribute to cancer progression by regulating tumor proliferation, invasion, and angiogenesis $(28,30)$. Inhibition of S1P-S1PR1 signaling has been considered a novel target for cancer therapy $(28,31)$. However, recently, Metodieva et al reported reduced expression of S1PRI in human NSCLC specimens compared to that in peripheral non-tumor tissues (32). On the other hand, receptor subtype-specific positive and negative regulation of S1P-S1PR1 signaling has been proposed. Furthermore, the direction of signaling is thought to depend on cell type $(33,34)$. In this study, expression of Edg family genes other than EDG1 was not significantly different between PC14HM cells and the parent cells (data not shown). Although further study is clearly necessary to validate the functional significance of the Edg family in lung cancer, it is possible that reduced expression of EDGl in lung cancer cells influences metastatic potential in lung cancer.

HS3ST3A1 encodes the enzyme 3-O-sulfotransferase, which catalyzes the biosynthesis of a specific subtype of heparan sulfate (HS), 3-O-sulfated heparan sulfate. This HS subtype has specific functional significance for herpes simplex virus-1 infection (35). To the best of our knowledge, no function of this molecule in cancer cells has previously been suggested. However, heparan sulfate cleavage by heparanase is strongly implicated in cell dissemination associated with tumor metastasis, angiogenesis, and inflammation (36). Upregulation of HS3ST3Al in PC14HM cells is thought to increase the biosynthesis of the specific HS subtype and to contribute to elevated metastatic potential. The function of HRB2 in human cells has not been determined.

Rab7 is a late endosome/lysosome-associated small GTPase, perhaps the only lysosomal Rab protein identified to date (37). Rab7 plays critical roles in the endocytic processes, participating in multiple regulatory mechanisms in endosomal sorting, the biogenesis of lysosomes, and phagocytosis. These processes are closely related to substrate degradation, antigen presentation, cell signaling, cell survival, and microbial pathogen infection. Consistently, mutations in or dysfunction of Rab7 result in traffic disorders, which cause various diseases, such as neuropathies, cancers, and lipid metabolism diseases (37). Frasa et al reported that Rab7 participated in the regulation of E-cadherin turnover and stability of cell-cell contacts by interacting with EGF and its downstream effector, Rac1 (38). In addition, Rab7-mediated lysosome trafficking is implicated in hepatocyte growth factor-induced invasion by prostate tumor cells (39). Elevated expression of $R A B 7$ in PC14HM cells and human cancer specimens may suggest a role of this molecule in carcinogenesis, cell adhesion, and invasion. Overall, the findings with these randomly selected differentially expressed genes may suggest that the panel of differentially expressed genes will contain functionally significant genes for metastasis in lung cancer.

In summary, we established a human lung cancer cell line with high metastatic potential to multiple organs from the $\mathrm{PC} 14$ cell line using an in vivo selection method. Using microarray analysis, we were able to identify a panel of genes that were differentially expressed between the highly metastatic subline and the parent cells. Although further studies are needed, as described above, these genes may contain candidates for molecular determinants of metastasis in lung cancer and may even provide candidate molecular targets for the treatment of metastatic lung cancer. This metastasis model system will be useful to further understand the molecular mechanism(s) of, and discover and characterize novel therapeutic strategies for, the metastasis of human lung cancer.

\section{Acknowledgements}

This study was supported, in part, by a Grant-in-Aid for Scientific Research (C) 17591457 from the Japan Society for the Promotion of Science (JSPS).

\section{References}

1. Minna JD, Roth JA and Gazdar AF: Focus on lung cancer. Cancer Cell 1: 49-52, 2002.

2. Sawabata N, Asamura H, Goya T, et al: Japanese Lung Cancer Registry Study: first prospective enrollment of a large number of surgical and nonsurgical cases in 2002. J Thorac Oncol 5: $1369-1375,2010$

3. Chaffer CL and Weinberg RA: A perspective on cancer cell metastasis. Science 331: 1559-1564, 2011.

4. Fidler IJ and Poste G: The 'seed and soil' hypothesis revisited. Lancet Oncol 9: 808, 2008.

5. Wirtz D, Konstantopoulos K and Searson PC: The physics of cancer: the role of physical interactions and mechanical forces in metastasis. Nat Rev Cancer 11: 512-522, 2011.

6. Yano S, Nishioka Y, Izumi K, et al: Novel metastasis model of human lung cancer in SCID mice depleted of NK cells. Int J Cancer 67: 211-217, 1996.

7. Kimura K, Nakano T, Park YB, et al: Establishment of human osteosarcoma cell lines with high metastatic potential to lungs and their utilities for therapeutic studies on metastatic osteosarcoma. Clin Exp Metastasis 19: 477-485, 2002.

8. Francia G, Cruz-Munoz W, Man S, Xu P and Kerbel RS: Mouse models of advanced spontaneous metastasis for experimental therapeutics. Nat Rev Cancer 11: 135-141, 2011.

9. Khanna $\mathrm{C}$ and Hunter $\mathrm{K}$ : Modeling metastasis in vivo. Carcinogenesis 26: 513-523, 2005.

10. Nagamachi Y, Tani M, Shimizu K, Tsuda H, Niitsu Y and Yokota J: Orthotopic growth and metastasis of human non-small cell lung carcinoma cell injected into the pleural cavity of nude mice. Cancer Lett 127: 203-209, 1998.

11. Jia D, Yan M, Wang X, et al: Development of a highly metastatic model that reveals a crucial role of fibronectin in lung cancer cell migration and invasion. BMC Cancer 10: 364, 2010.

12. Nakano T, Tani M, Ishibashi Y, et al: Biological properties and gene expression associated with metastatic potential of human osteosarcoma. Clin Exp Metastasis 20: 665-674, 2003. 
13. Yokota J: Tumor progression and metastasis. Carcinogenesis 21 : 497-503, 2000

14. Bittner M, Meltzer P, Chen Y, et al: Molecular classification of cutaneous malignant melanoma by gene expression profiling. Nature 406: 536-540, 2000.

15. Hashimoto Y, Shindo-Okada N, Tani M, Takeuchi K, Toma H and Yokota J: Identification of genes differentially expressed in association with metastatic potential of K-1735 murine melanoma by messenger RNA differential display. Cancer Res 56: 5266-5271, 1996.

16. Clark EA, Golub TR, Lander ES and Hynes RO: Genomic analysis of metastasis reveals an essential role for RhoC. Nature 406: 532-535, 2000

17. Hashimoto Y, Shindo-Okada N, Tani M, et al: Expression of the Elm1 gene, a novel gene of the $\mathrm{CCN}$ (connective tissue growth factor, Cyr61/Cef10, and neuroblastoma overexpressed gene) family, suppresses in vivo tumor growth and metastasis of K-1735 murine melanoma cells. J Exp Med 187: 289-296, 1998.

18. Raynaud CM, Mercier O, Dartevelle P, et al: Expression of chemokine receptor CCR6 as a molecular determinant of adrenal metastatic relapse in patients with primary lung cancer. Clin Lung Cancer 11: 187-191, 2010.

19. Bjorge JD, Pang AS, Funnell M, et al: Simultaneous siRNA targeting of Src and downstream signaling molecules inhibit tumor formation and metastasis of a human model breast cancer cell line. PLoS One 6: e19309, 2011.

20. Bulk E, Sargin B, Krug U, et al: S100A2 induces metastasis in non-small cell lung cancer. Clin Cancer Res 15: 22-29, 2009.

21. Hoffman RM: Orthotopic metastatic mouse models for anticancer drug discovery and evaluation: a bridge to the clinic. Invest New Drugs 17: 343-359, 1999.

22. Sawabata N, Miyaoka E, Asamura H, et al: Japanese lung cancer registry study of 11,663 surgical cases in 2004: demographic and prognosis changes over decade. J Thorac Oncol 6: 1229-1235, 2011.

23. Yano S, Shinohara H, Herbst RS, et al: Expression of vascular endothelial growth factor is necessary but not sufficient for production and growth of brain metastasis. Cancer Res 60: 4959-4967, 2000.

24. Arifin M, Tanimoto K, Putra AC, Hiyama E, Nishiyama M and Hiyama K: Carcinogenesis and cellular immortalization without persistent inactivation of $\mathrm{p} 16 / \mathrm{Rb}$ pathway in lung cancer. Int $\mathrm{J}$ Oncol 36: 1217-1227, 2010.

25. Noro R, Gemma A, Kosaihira S, et al: Gefitinib (IRESSA) sensitive lung cancer cell lines show phosphorylation of Akt without ligand stimulation. BMC Cancer 6: 277, 2006.

26. Lynch TJ, Bell DW, Sordella R, et al: Activating mutations in the epidermal growth factor receptor underlying responsiveness of non-small-cell lung cancer to gefitinib. N Engl J Med 350: 2129-2139, 2004.
27. Yeh HH, Ogawa K, Balatoni J, et al: Molecular imaging of active mutant L858R EGF receptor (EGFR) kinase-expressing non-small cell lung carcinomas using PET/CT. Proc Natl Acad Sci USA 108: 1603-1608, 2011.

28. Lee H, Deng J, Kujawski M, et al: STAT3-induced S1PR1 expression is crucial for persistent STAT3 activation in tumors. Nat Med 16: 1421-1428, 2010

29. Spiegel S and Milstien S: The outs and the ins of sphingosine1-phosphate in immunity. Nat Rev Immunol 11: 403-415, 2011.

30. Visentin B, Vekich JA, Sibbald BJ, et al: Validation of an antisphingosine-1-phosphate antibody as a potential therapeutic in reducing growth, invasion, and angiogenesis in multiple tumor lineages. Cancer Cell 9: 225-238, 2006.

31. Chae SS, Paik JH, Furneaux H and Hla T: Requirement for sphingosine 1-phosphate receptor-1 in tumor angiogenesis demonstrated by in vivo RNA interference. J Clin Invest 114: 1082-1089, 2004.

32. Metodieva SN, Nikolova DN, Cherneva RV, Dimova II, Petrov DB and Toncheva DI: Expression analysis of angiogenesis-related genes in Bulgarian patients with early-stage non-small cell lung cancer. Tumori 97: 86-94, 2011.

33. Yamaguchi H, Kitayama J, Takuwa N, et al: Sphingosine1-phosphate receptor subtype-specific positive and negative regulation of Rac and haematogenous metastasis of melanoma cells. Biochem J 374: 715-722, 2003.

34. Takuwa Y: Subtype-specific differential regulation of Rho family $\mathrm{G}$ proteins and cell migration by the Edg family sphingosine1-phosphate receptors. Biochim Biophys Acta 1582: 112-120, 2002.

35. Shukla D, Liu J, Blaiklock P, et al: A novel role for 3-O-sulfated heparan sulfate in herpes simplex virus 1 entry. Cell 99: 13-22, 1999.

36. Arvatz G, Shafat I, Levy-Adam F, Ilan N and Vlodavsky I: The heparanase system and tumor metastasis: is heparanase the seed and soil? Cancer Metastasis Rev 30: 253-268, 2011.

37. Zhang M, Chen L, Wang S and Wang T: Rab7: roles in membrane trafficking and disease. Biosci Rep 29: 193-209, 2009.

38. Frasa MA, Maximiano FC, Smolarczyk K, et al: Armus is a Rac1 effector that inactivates Rab7 and regulates E-cadherin degradation. Curr Biol 20: 198-208, 2010.

39. Steffan JJ, Williams BC, Welbourne T and Cardelli JA: HGF-induced invasion by prostate tumor cells requires anterograde lysosome trafficking and activity of $\mathrm{Na}^{+}-\mathrm{H}^{+}$exchangers. J Cell Sci 123: 1151-1159, 2010. 